\title{
Analyse und Förderung effektiver Lehr- Lernprozesse im Kontext evidenzbasierter Bildungsreform - Beiträge der Tübinger Forschergruppe für Empirische Bildungsforschung
}

\section{ZfE}

\author{
Katharina Scheiter · Josef Schrader · Ulrich Trautwein · Friedrich W. Hesse
}

Empirische Bildungsforschung hat drei zentrale Aufgaben: Sie hilft erstens bei der Diagnose von Kompetenzen der Lernenden bzw. der Funktionsfähigkeit des Systems. Neben der Diagnostik verfolgt sie zweitens das Ziel, die Ursachen für gelingende oder misslingende Lernprozesse und Bildungskarrieren zu identifizieren, sowohl auf der Ebene der Individuen und ihres unmittelbaren sozialen Umfelds als auch auf der Ebene von Lerngruppen, Bildungseinrichtungen und ihren übergreifenden Strukturen. Eine dritte Aufgabe der empirischen Bildungsforschung ist die Begleitung und Evaluation von Veränderungen im Bildungssystem und die Entwicklung und Prüfung von Interventionsmaßnahmen.

Im Jahr 2003 etablierte die Deutsche Forschungsgemeinschaft (DFG) eine Initiative zur Verbesserung der Situation der empirischen Bildungsforschung in Deutschland durch die Ausschreibung von Forschergruppen, die inhaltlich zu Themen der empirischen Bildungsforschung arbeiten und dabei auch strukturbildende Maßnahmen beinhalten sollten. Ziele dieser Initiative waren die wissenschaftliche Profilbildung an einer Hochschule, die Etablierung von Ausbildungsstrukturen in der empirischen Bildungsforschung, die Förderung

\footnotetext{
Online publiziert: 24.05 .2014

(C) Springer Fachmedien Wiesbaden 2014

Prof. Dr. K. Scheiter $(\bowtie) \cdot$ Prof. Dr. Dr. F. W. Hesse

Leibniz-Institut für Wissensmedien,

Schleichstraße 6, 72076 Tübingen, Deutschland

E-Mail: k.scheiter@iwm-kmrc.de

Prof. Dr. Dr. F. W. Hesse

E-Mail: f.hesse@iwm-kmrc.de

Prof. Dr. J. Schrader

Deutsches Institut für Erwachsenenbildung - Leibniz-Zentrum für Lebenslanges Lernen, Heinemannstr. 12-14, 53175 Bonn, Deutschland

E-Mail: schrader@die-bonn.de

Prof. Dr. U. Trautwein

Empirische Bildungsforschung und Pädagogische Psychologie, Universität Tübingen,

Europastraße 6, 72072 Tübingen, Deutschland

E-Mail: ulrich.trautwein@uni-tuebingen.de
} 
des wissenschaftlichen Nachwuchses auf diesem Gebiet, in geeigneten Fällen die Kooperation mit der Praxis sowie die Weiterentwicklung der empirischen Bildungsforschung.

Am Standort Tübingen erarbeiteten Wissenschaftlerinnen und Wissenschaftler des Leibniz-Instituts für Wissensmedien sowie der Universität (Institut für Erziehungswissenschaft und Fachbereich Psychologie) einen Antrag auf Einrichtung einer solchen Forschergruppe, die nach Bewilligung durch die DFG im Jahr 2007 unter dem Titel „Orchestrierung computerunterstützter Lehr-Lernprozesse“ ihre Arbeit aufnahm; ab 2010 erfolgte eine Umbenennung in „Analyse und Förderung effektiver Lehr-Lernprozesse“. Gegenstandsbereich der sich jetzt in der dritten und damit abschließenden Förderphase befindlichen Forschergruppe FOR738 bildet die Frage, wie organisierte Lehr-Lernprozesse analysiert und unterstützt werden können, wobei ein wesentliches (aber nicht ausschließliches) Augenmerk auf der Rolle digitaler Medien in diesem Kontext liegt.

Die Tübinger Forschergruppe, die im Sinne eines strukturbildenden Beitrages auch den Ausgangspunkt für die Etablierung des WissenschaftsCampus „Bildung in Informationsumwelten“ und der Graduiertenschule LEAD (Learning, Educational Achievement, and Life Course Development) bildete, zielt auf die Identifikation der Ursachen für effektive Lehr-Lernprozesse sowie auf die Entwicklung und Prüfung von Interventionsmaßnahmen bis hin zur exemplarischen Implementation (,small scaling up“). Der Fokus der Forschergruppe beschränkt sich hierbei auf Bedingungen wirkungsvoller Lehr-Lernprozesse, die von den unmittelbaren Akteuren beeinflussbar sind (bspw. durch den Lernenden selbst bzw. die Lehrperson).

Die Projekte der Tübinger Forschergruppe weisen folgende Spezifika auf: Im Sinne einer Nutzeninspirierung bildet die explizite Orientierung der Forschungsfragen an Herausforderungen der Praxis den Ausgangspunkt. Gleichzeitig werden durchgängig grundlagenwissenschaftliche Befunde zur Schärfung der Fragestellungen und zur Konzeptualisierung der empirischen Untersuchungen verwendet. Auf diese Weise wird generalisierbares Wissen angestrebt, das über die Evaluation und Optimierung lokaler Programme hinausgeht. Die Forschergruppe berücksichtigt die institutionellen Rahmenbedingungen, konzentriert sich jedoch auf organisierte Lehr-Lernprozesse. Um die bearbeiteten Forschungsfragen zu verschiedenen Facetten und Einflussfaktoren, die für organisierte LehrLernprozesse bedeutsam sind, zu verorten, wird auf das Angebots-Nutzungs-Modell von Helmke (2012) zurückgegriffen. Dieses Modell beschreibt organisierte Lehr-Lernprozesse (Unterricht) in integrativer Weise sowohl auf einer Prozess- als auch einer Produktebene und erlaubt eine Benennung und Kontextualisierung der relevanten Wirkfaktoren auf verschiedenen Betrachtungsebenen. Bei Unterricht handelt es sich danach um ein von einer Lehrperson gemachtes Angebot, welches durch die lernende Person wahrgenommen und interpretiert wird. Diese Interpretation bedingt unterschiedliche Lernaktivitäten (Nutzung), die zusätzlich durch individuelle Voraussetzungen der Lernenden (Lernerpotenzial) mit beeinflusst werden und die sich auf den Lernerfolg auswirken. Familiäre, soziale und strukturelle Kontextfaktoren beeinflussen dieses komplexe Wirkungsgefüge. Entsprechend konzentrieren sich die Forschungsfragen der Tübinger Forschergruppe darauf, welche individuellen Eingangsmerkmale und kognitiven Aktivitäten der Lernenden am Wissens- und Kompetenzaufbau beteiligt sind und wie dieser durch entsprechende Interventionen begünstigt werden kann. Als methodische Herangehensweise wird ein (quasi-) experimenteller Zugang favorisiert, um möglichst belastbare Aussagen zu der Wirkung bestimmter Determinanten zu erhalten. Zusammenfassend liegt der inhaltliche Fokus der 
Forschergruppe auf der Identifizierung effektiver Lernaktivitäten, deren Voraussetzungen und deren Förderung unter Berücksichtigung der Potenziale digitaler Medien.

Mit der Konzentration auf effektive Lernaktivitäten wird der Tatsache Rechnung getragen, dass erfolgreiches Lernen das Ergebnis einer aktiven Auseinandersetzung mit den Lerninhalten ist, die die Wahl und das Verfolgen von Zielen, die Regulation von Ressourcen sowie die Anwendung kognitiver und metakognitiver Lernstrategien umfasst (z. B. Boekaerts 1999; Zimmerman 2000; Schmitz 2001). Eine die Arbeit der Forschergruppe leitende Auffassung ist, dass die Ausführung effektiver Lernaktivitäten an bestimmte Voraussetzungen gebunden ist. Sie hängt einerseits ab von individuellen Merkmalen der Lernenden (dem Lernpotenzial sensu Helmke 2012). Neben diesem Lernpotenzial sind andererseits spezifische Merkmale des Unterrichts wesentliche Voraussetzungen für die Ausführung effektiver Lernaktivitäten. Effektive Lernaktivitäten werden oftmals nicht spontan ausgeführt; vielmehr bedürfen sie einer instruktionalen Förderung. Zentrales Anliegen der Forschergruppe besteht daher darin, Interventionen zu entwickeln, die eine Intensivierung und Optimierung der Ausführung wirkungsvoller Lehr-Lernaktivitäten bewirken. Die Potenziale digitaler Medien spielen in vielen Projekten der Forschergruppe eine zentrale Rolle, indem sie einerseits Gegenstand der Forschung und andererseits wesentlich für die Umsetzung der Interventionsansätze sind.

Der hier vorgelegte Thementeil enthält ausgewählte, exemplarische Studien aus dem multi- und interdisziplinären Umfeld der Tübinger Empirischen Bildungsforschung. Zunächst wird im Stichwortartikel von Schrader die Diskussion um das Prinzip der Evidenzbasierung in der nationalen und internationalen Debatte zur Empirischen Bildungsforschung der vergangenen Jahre nachgezeichnet. Diese Diskussion bildet den thematischen und methodischen Hintergrund für die forschungsstrategische Ausrichtung der Tübinger Gruppe, die in mittelfristig angelegten Forschungsprojekten Anwendungs- und Grundlagenorientierung zu verknüpfen sucht.

Im Beitrag von Scheiter, Schüler, Gerjets, Stalbovs, Schubert und Hesse wird die Rolle aufgabenspezifischer und fachspezifischer Motivationskomponenten für den Wissensaufbau beim Lernen mit Text versus Multimedia adressiert. Motivation spielt eine wesentliche Rolle für die Ausführung kognitiver Lernaktivitäten, indem sie die Anwendung (vorhandener) Verarbeitungsstrategien begünstigt. In dem Beitrag wird in einer feldexperimentellen Studie untersucht, inwieweit und auf welche Weise die Lerneffektivität von Text bzw. Multimedia differenziell durch die Eingangsmotivation der Lernenden moderiert wird.

Der Beitrag von Schreier, Dicke, Gaspard, Häfner, Flunger, Lüdtke, Nagengast und Trautwein konzentriert sich ebenfalls auf die fachspezifische Motivation, und zwar im Hinblick auf deren Entstehungsbedingungen im Unterrichtskontext. Vor dem Hintergrund des Erwartungs-Wert-Modells der Motivation (Eccles et al. 1983) werden Unterrichtsstrategien, die die Relevanz mathematischer Lehrinhalte betonen (z. B. Praxisorientierung, Themeneinführung mit Alltagsbeispielen), mit den Wertüberzeugungen der Schülerinnen und Schüler in Zusammenhang gesetzt. Auch die Rolle der fachspezifischen Wertschätzung im Klassenverband wird im Hinblick auf Schülermotivation untersucht.

Die anschließenden zwei Beiträge widmen sich explizit der Frage, wie Wissens- und Kompetenzaufbau vermittelt durch die Ausführung kognitiver Lernaktivitäten durch entsprechende Interventionen gefördert werden können. Die Interventionen nutzen dabei die Potenziale digitaler Medien in spezifischer Weise. Im Beitrag von Hetfleisch, Goeze und Schrader wird die mikrodidaktische Implementation eines in (quasi-)experimentel- 
len Vorgängerstudien entwickelten und kompetenzfördernden didaktischen Konzepts in die alltägliche Lehrerausbildungspraxis untersucht. Das Konzept umfasst eine digitale Lernumgebung, die durch didaktisch angereicherte Videofälle die Entwicklung der Kompetenz (angehender) Lehrender zur Diagnose von Lehr-Lernsituationen zielgerichtet unterstützt. Geprüft wird in einer quasi-experimentellen Feldstudie, wie es sich auf diese ursprünglich intendierte Wirksamkeit des Konzepts auswirkt, wenn Lehrerausbilder selbst die videofallbasierte Intervention in ihren Referendarskursen einsetzen - und zwar unter variierten Graden pädagogischer Autonomie.

Schließlich wird im Beitrag von Link, Huber, Schwarz, Fischer, Nuerk, Cress, und Moeller untersucht, inwieweit der Einsatz einer digitalen Tanzmatte im Rahmen eines verkörperlichten Trainings basisnumerischer Repräsentationen von Grundschulkindern einen positiven Einfluss auf deren Verständnis der Platz x Wert-Struktur des arabischen Zahlsystems hat. In der feldexperimentellen Evaluationsstudie werden dabei verschiedene Interventionsvarianten miteinander verglichen, um die Spezifität der erwarteten Trainingseffekte genauer beschreiben zu können.

Die hier präsentierten Arbeiten wären nicht möglich gewesen ohne die mehrjährige Förderung durch die Deutsche Forschungsgemeinschaft. Dafür sind wir sehr dankbar. Dankbar sind wir auch für die Sammelrezension, die Peter Drewek zu neueren Veröffentlichungen zur Empirischen Bildungsforschung verfasst hat und die (uns) hilft einzuschätzen, was noch zu tun bleibt für eine empirische Bildungsforschung, die auf die Analyse und Förderung effektiver Lehr-Lernprozesse zielt.

\section{Literatur}

Boekaerts, M. (1999). Self-regulated learning: Where we are today. International Journal of Educational Research, 31, 445-457.

Eccles, J. S., Adler, T. F., Futterman, R., Goff, S. B., Kaczala, C. M., Meece, J. L., \& Midgley, C. (1983). Expectancies, values and academic behaviors. In J. T. Spence (Hrsg.), Achievement and achievement motives: Psychological and sociological approaches (S. 75-146). New York: Freeman.

Helmke, A. (2012). Unterrichtsqualität und Lehrerprofessionalität. Diagnose, Evaluation und Verbesserung des Unterrichts (4. Aufl.). Seelze: Klett-Kallmeyer.

Schmitz, B. (2001). Self-Monitoring zur Unterstützung des Transfers einer Schulung in Selbstregulation für Studierende: Eine prozessanalytische Untersuchung. Zeitschrift für Pädagogische Psychologie, 15, 181-197.

Zimmerman, B. J. (2000). Attaining self-regulation: A social cognitive perspective. In M. Boekaerts, P. R. Pintrich, \& M. Zeidner (Hrsg.), Handbook of self-regulation (S. 13-39). San Diego: Academic Press.

\section{Anmerkung}

Die im vorliegenden Themenheft vorgestellten Studien wurden im Rahmen der durch die Deutsche Forschungsgemeinschaft geförderten Forschergruppe FOR738 „Analyse und Förderung effektiver Lehr-Lernprozesse“ durchgeführt. 